\title{
Molecular Dynamics Study on Structural and Atomic Evolution Between Au and Ni Nanoparticles Through Coalescence
}

\section{Bangquan Li}

Shanxi Datong University

Jing Li

Shanxi Datong University

Xiaoqiang Su

Shanxi Datong University

Yimin Cui ( $\nabla$ cuiym@buaa.edu.cn )

Beihang University

\section{Research Article}

Keywords: Molecular Dynamics, Nanoparticles, Coalescence

Posted Date: May 11th, 2021

DOI: https://doi.org/10.21203/rs.3.rs-500450/v1

License: (c) This work is licensed under a Creative Commons Attribution 4.0 International License.

Read Full License

Version of Record: A version of this preprint was published at Scientific Reports on July 29th, 2021. See the published version at https://doi.org/10.1038/s41598-021-94822-0. 


\section{Abstract}

Motivated by the structure evolution experiments of Janus NiAu nanoparticles (NPs), we present a detailed study on the thermodynamic evolution of Ni and Au NPs with different ratios of Au and Ni through the molecular dynamics (MD) simulations. It is found that, for fixed Ni particle size $(5.8 \mathrm{~nm}$ in diameter), the energy variation with the increasing temperature is related to the Au sizes (1.5-9.6 nm in diameter), due to the diverse atomic segregation modes. For a small Au particle, due to lattice induction, the structure will change from order to disorder and then to order. The interface defects of the merging NPs could be automatically eliminated by coalescence processes. The change in energy as the temperature increases is similar to that of monometallic NPs. For larger Au particles, the irregular variation of energy occurs and the atomic energy experience one or two reductions with the increase of the temperature. The segregation of Au atoms to the surface of Ni particle is dominant during the continuous heating process. The coalescence processes of Au atoms strongly determine the final morphology of the particles. Dumbbell-like, Janus and eccentric core-shell spherical structures could be obtained during the heating process. Our results will provide an effective approach to the design of novel materials with specific properties through thermal control.

\section{Introduction}

It is well known that the physical and chemical properties of metallic nanomaterials depend strongly on their size and morphology ${ }^{1,2}$. Heterogeneous bimetallic nanoparticles (NPs) consisting of magnetic and noble metals possess enhanced magnetic, optical, and catalytic performance compared to the corresponding monometallic NPs ${ }^{3,4}$. In addition, the designing of the spatial arrangement and interfacial structure of NPs could further promote performance optimization and reveal structure-property relationship. Coalescence is an important growth mode in particle formation processes ${ }^{5,6}$. Coalescence of NPs and the evolution of their shapes have been widely investigated in recent years. For instance, the coalescence of decahedral gold NPs was investigated with the in-situ TEM technique, and the faceted structure of NPs was found to play a crucial role in agglomeration and coalescence ${ }^{7}$. As soft particles, when the silver NPs came into contact and underwent a spontaneous coalescence process, they could form the conductive films ${ }^{8}$. The metastable structures of bimetallic Ag-Cu NPs, such as Janus or Ag@Cu core/shell, were produced after coalescence below the melting point ${ }^{9}$. In-situ TEM images showed that Ostwald ripening process was found to be more prevalent at higher temperature during the sintering process of $\mathrm{Ag}$ and $\mathrm{Pt} \mathrm{NPs}{ }^{10}$. Molecular dynamics (MD) simulations revealed that coalescence of iron nanoclusters occurred at a temperature lower than the melting point of the clusters, and the difference in temperatures between coalescence and melting increased as the decreasing of cluster size ${ }^{11}$. The collision and coalescence of gold clusters indicated that the morphology and structure of the final particle were determined strongly by the coalescence processes ${ }^{12}$. The energy decrease was mainly caused by the reduction of surface energy occurred with coalescence of $\mathrm{Ag}$ and Pd clusters. The Pd atoms preferred staying inside the cluster with $\mathrm{Ag}$ coming to the surface ${ }^{13}$. The sintering procedure of FeNi NPs resulted in phase-segregated particles that are comprised of Fe-enriched surface and Ni- 
enriched core ${ }^{14}$. In our previous work, the Au-Ni Janus NPs were synthesized in a mild chemical solution system. The structure evolution behaviors were studied using an in-situ Cs-corrected STEM method at atomic scale ${ }^{15}$.

The understanding of coalescence processes may have lots of potential applications in finding optimal approaches to control the size and shape of the particles. However, the structure evolution derived mainly from surface atoms, which only reveals the composition of the surface layer, cannot identify the atom evolution of the inner core or interfaces. On the other hand, magnetic metal Ni have smaller atom size and higher surface energy compared to the noble metal $\mathrm{Au}$, rendering the immiscibility under equilibrium conditions with a large lattice mismatch (e.g., 15.7\% between Ni (3.524 $)$ ) and Au (4.079 ̊) with a face centered cubic (FCC) structure $)^{16}$. Benefitting from synergistic effect, it is reasonable to design and control Au-Ni structures by engineering the size, composition and atomic configuration in order to acquire distinctive properties.

The MD simulations with classical potentials method is known to be a tool of great reliability in the study of structural evolution and dynamic information of NPs ${ }^{17-19}$. In this work, we carried out a comparative study of segregation characteristics of coalescence process between Au and Ni NPs by MD simulations. Our results will provide both an effective approach to design novel materials with specific properties by thermal controlling and a perspective on the application of bimetallic NPs in high temperature environments.

\section{Methods}

The embedded atom method (EAM) was adopted to describe the interatomic interaction of Au-Ni. In the EAM force field ${ }^{20}$, the potential parameters were optimized according to basic material properties such as lattice constants, elastic constants, bulk moduli and vacancy formation energies, which provide a reasonable approximation to the interactions between different metal elements. In a bimetallic system, the total potential has been successfully applied previously 21,22 .

Our MD simulations are performed with large-scale atomic/molecular massively parallel simulator (LAMMPS) developed by Sandia National Laboratories ${ }^{23}$. The MD simulations were performed in the canonical NVT (fixed number of particles, volume and temperature) ensemble using the Nose-Hoover thermostat for maintaining the temperature. Typically, the equations of motion were integrated using the velocity-Verlet integrator with a time step of $0.001 \mathrm{ps}$.

As shown in Fig. 1, spherical gold and nickel NPs are extracted from a bulk FCC crystal with the [100], [010] and [001] crystallographic directions along the $x, y$-and $z$-axes, respectively. A perfect FCC lattice structure was constructed as the initial model with the corresponding lattice constants being $3.524 \AA$ and $4.079 \AA$ for $\mathrm{Ni}$ and $\mathrm{Au}$, respectively. As initial simulated objects, Au-Ni structures contained variable $\mathrm{Au}$ atoms (from 96 to 27311) and $9092 \mathrm{Ni}$ atoms. That is, for each pair, Ni nanoparticle has the same diameter of $5.8 \mathrm{~nm}$ and the diameter of Au nanoparticle is varied, ranging from $1.5 \mathrm{~nm}$ to $9.6 \mathrm{~nm}$ (shown 
in the Table S1). The corresponding eight different concentrations are considered to be $\mathrm{Au}_{0.01} \mathrm{Ni}_{0.99}$ (NP1), $\mathrm{Au}_{0.05} \mathrm{Ni}_{0.95}$ (NP2), $\mathrm{Au}_{0.1} \mathrm{Ni}_{0.9}$ (NP3), $\mathrm{Au}_{0.25} \mathrm{Ni}_{0.75}$ (NP4), $\mathrm{Au}_{0.4} \mathrm{Ni}_{0.6}$ (NP5), $\mathrm{Au}_{0.5} \mathrm{Ni}_{0.5}$ (NP6), $\mathrm{Au}_{0.6} \mathrm{Ni}_{0.4}(\mathrm{NP7})$ and $\mathrm{Au}_{0.75} \mathrm{Ni}_{0.25}$ (NP8). Au and Ni NPs are placed with one another (along the $x$ axis), at a distance equal to the length of $\mathrm{Ni}$ lattice constant.

Prior to starting the MD simulations, the NPs system was relaxed thoroughly at $0.1 \mathrm{~K}$ until the system reaches a steady state, then these NPs were subjected to a continuous heating with the heating rate of $0.2 \mathrm{~K} / \mathrm{ps}$ in each simulation when the temperature increased from $0.1 \mathrm{~K}$ to $2100 \mathrm{~K}$, and lasting $300 \mathrm{ps}$ relaxation at $2100 \mathrm{~K}$, which is far above the melting point of bulk Au and Ni, so as to melt the whole NPs.

\section{Simulation Results And Discussion}

By slightly heating the configurations, we can get insight into the shape changes and melting processes of such congeries. In a heating process, the two NPs are drawn together quickly by the excess surface energy. Subsequently, two NPs constantly change their positions, looking for state in space in order to find a lowest energy configuration ${ }^{24}$. Figure 2 shows snapshots of the NP1 during the simulation process. The two NPs are initially separated without heating applied yet (in Fig. 2a), and the atoms arrangements of the (100) of Au and Ni are shown in Fig. 2b and Fig. 2c. Before the coalescence of the Au atoms of NP1, the particles undergo the shape convulsion which has been termed "quasimelting", and then coalesce into the large one at a highly accelerated rate compared with its prior migration. The approach and rotation of the two NPs can be clearly seen in the Supporting Information, Video S1. The small Au particle start heating, Au atoms and $\mathrm{Ni}$ atoms of (100) face hold atomic arrangement FCC structure (Fig. 2(b) and (c)), During heating process, the pair distribution function (PDF) of Au atoms and $\mathrm{Ni}$ atoms are also employed to monitor particle structure evolutions during the simulation. As shown in Fig. 2(d), only first-nearest neighbor peaks of Au atomic arrangement can be seen in the PDF of the NP1 at 10K, and the crystalline peaks disappear, while the FCC structure is restored at $100 \mathrm{~K}$. When the temperature is less than 100K, Au NPs and Ni NPs did not contact. The Au NPs transform or rotate in space in order to find a low-energy configuration ( Au NPs are not the FCC) and then Au and Ni NPs are drawn together quickly by the excess surface energy, which is similar to the experiment was observed ${ }^{25}$. Subsequently, when the nanoparticle is small, its atoms are incorporated into the structure until almost every atom of the nanoparticle has rearranged over the (100) surface of the Ni NPs. We have studied the same size of the single element Au without $\mathrm{Ni}$ conditions, in which the Au structure has not changed in the same temperature. That is to say, the coalesced clusters with larger Ni NPs form an FCC structure. Structural transformation was induced by NPs containing greater numbers of atoms ${ }^{26}$.

Au atoms are induced by $\mathrm{Ni}$ atoms lattice, and the cross section of $\mathrm{Au}$ atoms can also illustrate this point. As shown in the Fig. 3, when the temperature is $6 \mathrm{~K}$, the Au atoms of NP1 are arranged in an orderly manner. When the temperature is $10 \mathrm{~K}$, the structure is deformed, and at a temperature of $100 \mathrm{~K}$, Au atoms restore the ordered structure. We also found that in the process of merging, the interface defects disappeared in Fig. 3. When the temperature is $94 \mathrm{~K}$, Au and Ni start to contact, and soon Au atoms in 
accordance with $\mathrm{Ni}(100)$ arrangement, the defects were observed during the process. As the temperature increases, the defects are gradually shifted and eliminated. These results allowed us to identify the remaining defects are originated from the coalescence phenomenon mentioned previously, which can be eliminated by means of energy input into the system. The eliminate process can be clearly seen in the Supporting Information, Video S2. During the coalescence process, the surface atoms showed "flow" characteristics and "flowed" toward the neighboring Au NP because of the atomic interaction forces between the Au NPs ${ }^{27}$. On the other hand, the NPs tend toward a more stable structure, displacing defects and releasing internal stresses ${ }^{28}$. These findings suggested that thermal energy could be applied to reduce the number of coalescence defects, which might cause a poor performance. It is also found that, with the increasing of Au atoms, Au atoms and $\mathrm{Ni}$ atoms interface are still ordered. We believe that no matter how many the number of Au atoms, Ni content will induce Au in an orderly arrangement over the (100) surface due to Au atoms more fluidity.

To analyze the stability and structural transformation of Ni-Au NPs, the caloric curves of potential energy variation on temperature is illustrated in Fig. 4. The caloric curve of metallic nanomaterials is very different from that of bulk materials due to the decrease in the system size. The transition is no longer sharp but smooth and takes place over a finite temperature range ${ }^{29}$. The melting point corresponding to the temperature value is determined by monitoring the change where there is a jump in the slope of the caloric curves. The sudden increase in energy over a small temperature change indicates that the first order transition from solid to liquid phase is similar to previous work ${ }^{22}$. Figure $4 a$ shows the caloric curves calculated from the NP1, NP2, NP3 and NP4. The NP1 shows a well-defined melting transition upon heating in the caloric curve. The change in energy of NP1 as the temperature increases is similar to that of monometallic NPs. For the NP2, NP3 and NP4, there is an irregular phenomenon of the melting that the energy decreases with the increase of the temperature. This is because that the Au atoms have lower surface energy, and the distribution of Au atoms on the surface layer can make the NPs in the state of low free energy. There is a competition between the decreased energy induced by the atomic segregation and the increased energy as the temperature increases. Then the atomic energy increases with the increase of the temperature. The sudden increase of the energy indicates that the total NPs melt. For NP5 in Fig. 4b, the irregular phenomenon occurs twice in the melting process. When the number of Au atoms increases, the Au atomic segregation leads to the decrease in the potential energy. After staying in a certain temperature, Au atoms will melt and release latent heat. When the energy increase in the temperature is more than the energy decrease induced by the atomic segregation, the first jump occurs. At this time Ni NPs has not melted yet, Au atoms continue to wrap Ni NPs, repeating the energy reduction process until Ni melts, then occurring a second jump. Though, relative to sample NP5,the first energy decrease of NP6, NP7 and NP8 is not obvious, still undergoing the same structure change process.

To better understand the nature of the energy change, we select the cross section of the particle at temperature 1173K in detail. As shown in Fig. 5a, Au atoms segregated to the surface of Ni particle is dominant during the continuous heating of NPs. The Au particles have melted and Ni particles are not melted. For NP1, NP2, NP3, Au atoms diffused along the surface of Ni. For NP4, NP5, NP6, Au and Ni 
form alloying at the interface besides the diffusion. This implies that Au and Ni can form alloy NPs although they are not mismatched in the bulk ${ }^{30,31}$. When Au is large enough (NP7, NP8), Au atoms encapsulate completely due to Au atoms segregation. With the Au atoms "flowing", we can get the eccentric core-shell structure Au@Ni NPs. As shown in Fig. 5b and 5c, the PDF peaks reflect the local order of structure. The first PDF peak location on the curve corresponds to the nearest interatomic distances and the second peak corresponds to the lattice parameter. The Au atomic PDF of structure obtained at $1173 \mathrm{~K}$ are shown in Fig. 5b, and the corresponding PDF at $0.1 \mathrm{~K}$ are shown in Fig. S1a. There is only the first nearest-neighbor peak relative to the PDF at $0.1 \mathrm{~K}$, and the other primary peak of FCC structures disappear. This indicates that PDF of Au atoms displays the transition from ordered structure to amorphous structure. The Ni atomic PDFs at $1173 \mathrm{~K}$ and $0.1 \mathrm{~K}$ are shown in Fig. $5 \mathrm{c}$ and Fig. S1b. As shown in Fig. $5 \mathrm{c}$, the second peak position on the curve corresponding to the Ni particle lattice parameter, is found to be $0.355-0.365 \mathrm{~nm}$, which is more than that of $0.1 \mathrm{~K}(0.351 \mathrm{~nm})$. On the other hand, the width of the peak becomes wider. All characteristic peaks still occur. This illustrates that the Ni particles has similar characteristics (FCC structure) at $1173 \mathrm{~K}$ and $0.1 \mathrm{~K}$. These observations also suggest that diverse melting modes occur during the continuous heating of Au-Ni NPs.

In order to obtain an intuitive size change of atomic diffusion in these Au-Ni NPs during the continuous heating, the concept of statistical radius was introduced into this work ${ }^{32}$. It can be seen from Fig. $6 \mathrm{a}$ and $6 \mathrm{~b}$ that the statistical radii of Au and Ni NPs slowly increases with increasing temperature at low temperatures, which is usually attributed to the thermal expansion of lattice for the Au and Ni contents in the Au-Ni NPs. With the temperature further rising, the statistical radius abruptly increases with a bigger slope indicating the solid-liquid transition. Moreover, it can be noted from the curves that beyond the melting points, the statistical radii of Au are gradually decreased which is also caused by miscible between $\mathrm{Au}$ and $\mathrm{Ni}$ atoms. As shown in Fig. 6b, it can be noted that the statistical radii curves of $\mathrm{Ni}$ particles are obviously divided into two cases despite the same size of Ni particles. For NP1, NP2 and NP3, the radii of Ni particles become larger at high temperature, indicating that Ni particles require higher temperatures to deform. It is possible that the alloying only takes place on the surface because Au atoms only diffuse to the surface of $\mathrm{Ni}$ with a lower fraction of Au atoms in the NPs. Alloying between interior Ni atoms and Au atoms becomes almost impossible. For larger particle from NP4 to NP8, the transition is more apparent and occurs at low temperature. The reason could be that Au particles of larger size can simultaneously alloyed with $\mathrm{Ni}$ at the interface accompanied the diffusion process, which leads to the increase of the radius of $\mathrm{Ni}$. To study the atomic arrangement of $\mathrm{Au}$ and $\mathrm{Ni}$, volume increments of $\mathrm{Ni}$ and $\mathrm{Au}$ particles before and after melting are plotted in Fig. 6c. It can be seen that the ratio of $\mathrm{Au}$ and $\mathrm{Ni}$ atoms is 1:1, and the volume increment is the same. The reason could be that $\mathrm{Au}$ and $\mathrm{Ni}$ are miscible after melting. The mean square displacement (MSD) is a measure to describe the average distance that a particle travels in the sintering process. Figure $6 \mathrm{~d}$ shows the MSD for the eight pairs of particles under the heating process. The MSD departs from the almost zero value and increases significantly, indicating the occurrence of atomic motion or melting. In general, when a small particle coalesces with a larger one, the temperature effect helps sintering where the smaller NPs tends to be incorporated into the larger ones ${ }^{33}$. The coalescence research of different sized gold NPs indicates that the melting occurs earlier when the 
size of the second particle is close to the first one ${ }^{34}$. The studies do not consider the weight of atoms due to the same element. However, as shown by the arrow in Fig. $6 \mathrm{~d}$, it is obvious that the atomic motion of Au-Ni NPs occurs earlier when the weights of Au and Ni particles are nearly the same according to our calculation (The NP4 being a typical representative). To perform an in-depth structural study, the MSD of $\mathrm{Ni}$ and $\mathrm{Au}$ atoms from NP1-NP8 are shown in the Fig. S2. The lighter particle tends to move towards heavier one. This illustrates that Au has a stronger affinity capacity relative to Ni when the size of $\mathrm{Au}$ particles is equal to that of $\mathrm{Ni}$ ones during the coalescence processes. The MSD of $\mathrm{Ni}$ and $\mathrm{Au}$ atoms almost experience a sharp rise simultaneously with the temperature increase, as can be seen from Fig. S2(d). Our atomic-scale observations clearly reveal that the weight plays a significant role during the process and the coalescence is driven by the surface diffusion and interface alloying. It can also be seen from Fig. S2 that the MSD from NP4 to NP8 provides direct evidence which indicates that Ni atoms are moved towards Au atoms.

To further investigate the change in morphology of the Au-Ni NPs in a quantitative way, the average

distance $D_{\text {ave }}$ between the $\mathrm{Au} / \mathrm{Ni}$ atoms and the NPs center of coordinate is

$$
D_{\text {ave }}=\frac{1}{N} \sum_{1}^{N}\left|r_{i}-r_{C}\right|
$$

where $r_{i}$ is the position of the $i$ atom and $r_{C}$ is the center of coordinate. The temperature dependence of this amount was obtained by calculating $D_{\text {ave }}$ at each temperature ${ }^{11}$. $D_{\text {ave }}$ is sensitive to the shape of the Au-Ni NPs, and has a maximum value when the Au NPs and Ni NPs are separated and decreased when the NPs coalesce. A minimum value of $D_{\text {ave }}$ is obtained for spherical Au-Ni NPs, which is the shape of some solid phase clusters or the liquid clusters. Here $D_{\text {ave }}$ is employed to describe the coalescence process. According to the segregation of $\mathrm{Au}$ and the melting of $\mathrm{Ni}$, the evaluation of $D_{a v e}$ of the NPs can be divided into three stages as shown in Fig. 7. Meanwhile, representative atomic morphology of the five representative temperatures of NP1-NP8 (in Table S2) is shown in Fig. S3. With respect to the behavior of the coalescence process, the dumbbell-like, Janus and eccentric core-shell spherical structure could be obtained during heating process.

\section{Dumbbell-like}

The first stage corresponds to the process from separate to contact state (Am points, $m=1-8$ ) between Au particles and Ni particles, the sample changes are similar from NP1 to NP8, and the $D_{\text {ave }}$ of NPs decreases sharply once the heating simulation starts. Then, the $D_{a v e}$ slightly increases or remains almost unchanged. This is because when the sintering neck was growing, the barycenter of system keep stable in the low temperature. The NPs are mainly in the form of dumbbell.

\section{Janus-like NPs}

After the coalesce process, the value of $D_{\text {ave }}$ decreases as stage $2(\mathrm{Am}$ to $\mathrm{Cm}, \mathrm{m}=1-8)$. The $D_{\text {ave }}$ of NP1 rapidly decreases at the point $A 1$ due to the small number of Au atoms in NP1 and the rapid diffusion to 
the surface of Ni NPs. The $D_{\text {ave }}$ of NP2 and NP3 decreases at point A2 and A3, and then slowly rises, but after that drops to the lowest point $\mathrm{C} 2$ and $\mathrm{C} 3$. As shown in the Fig. 7b, the $D_{\text {ave }}$ of NP4, NP5 and NP6 continue to decrease from point $A 4, A 5$ and $A 6$ to the lowest point $C 4, C 5$ and $C 6$ respectively. This suggests that as the number of $\mathrm{Au}$ atoms increases, Au gradually diffuses to the $\mathrm{Ni}$ surface until a spherical structure is formed. And for NP7 and NP8, the most interesting phenomenon is that the $D_{\text {ave }}$ decreases suddenly from $\mathrm{B} 7$ and $\mathrm{B} 8$ points to $\mathrm{C} 7$ and $\mathrm{C} 8$ points respectively. This indicates that the number of Au atoms is sufficient, and Ni NPs can be suddenly adsorbed into the Au NPs.

\section{The eccentric core shell NPs}

The stage 3 is corresponding to the melting process of Ni NPs (Dm points to Em points $(m=1-8)$ in Fig. 7.). The $D_{a v e}$ of NP1 to NP6 increases significantly with the rising temperature, which is corresponding to point $\mathrm{C} 1, \mathrm{C} 2, \mathrm{C} 3, \mathrm{C} 4, \mathrm{C} 5$ and $\mathrm{C} 6$ to point D1, D2, D3, D4 D5 and D6 respectively as shown in Fig. $7 \mathrm{a}$ and Fig. 7b. This is mainly due to the increasing temperature of the larger atomic spacing of Ni. In Fig. 7c, because $D_{a v e}$ does not reach the minimum points $C 7$ and $C 8$, the $D_{a v e}$ decreases slowly from points $\mathrm{C} 7$ and $\mathrm{C} 8$ points to $\mathrm{D} 7$ and $\mathrm{D} 8$ point respectively. This is due to the continuous wrapping process when $\mathrm{Ni}$ atoms are adsorbed inside Au particles. From Fig. S3, we can find that the NPs form a nonconcentric structure for NP7 and NP8.

The morphology evolution in Fig. S3 conforms to the change of $D_{\text {ave }}$ (maximum value means separate state, and minimum value represents the formation of a sphere). As can be seen in Fig. S3, Au atoms preferentially diffuse to the Ni surface ${ }^{9,22}$, so Au and Ni combine to form dumbbell, Janus and eccentric core shell structure. The in-situ TEM experimental results of structure evolution behaviors of NiAu nanospindles shows that Au component wraps along Ni matrix to form a core-shell like structure at promoted temperature ${ }^{15}$. The conclusions directly verify our simulation results. The catalytic activity is highly dependent on the evolution of alloying and phase segregation ${ }^{35,36}$. The thermal control of the nanoscale alloying and phase segregation is of practical importance for its application as a catalyst and design of nanostructures.

\section{Conclusions}

Using MD simulations, we have studied coalescence on Au-Ni NPs. Ni NPs has the same diameter of 5.8 $\mathrm{nm}$ and the Au NPs diameter ranged from $1.5 \mathrm{~nm}$ to $9.6 \mathrm{~nm}$. We have investigated the contributions to the NPs coalescence process. Au atoms progressively diffuse to the surface of Ni NPs, which are in agreement with the existing TEM observations, and the alloying and segregation processes were found in these NPs. For small Au particles, the structure of sequence will change due to the lattice induction, and coalescence processes eliminate the interface defects of the NPs. With the increasing temperatures, change energy of small Au particles was similar as monometallic NPs, but for larger Au particles, the irregular variation of energy occurs and the atomic energy experiences one or two reductions. The weight ratio plays a primary role for relative motion between $\mathrm{Au}$ and Ni particles. In addition $\square$ this study indicates 
that the formation of various shapes of NPs, dumbbell, Janus and non-concentric spherical structure can be tuned by the coalescence temperature and Au content. When the number of $\mathrm{Au}$ atoms is sufficient, $\mathrm{Ni}$ NPs can be suddenly adsorbed into the Au NPs to form an eccentric core-shell spherical structure.

\section{Declarations}

\section{Acknowledgements}

This work was supported by the National Natural Science Foundation of China (Nos. 61805129), the Science and Technology Project of Shanxi province (No.201801D221161).

\section{Author contributions}

Bangquan Li conducted MD simulations, analyzed the results, and wrote the manuscript. Jing Li conceived the research idea and constructed the models. Xiaoqiang Su defined the problem and reviewed the final manuscript. Yimin Cui analyzed the results and revised the manuscript critically.

\section{References}

1. Padilla-Cruz, A. L. et al. Synthesis and design of Ag-Fe bimetallic nanoparticles as antimicrobial synergistic combination therapies against clinically relevant pathogens. Sci. Rep. 11, 5351 https://doi.org/10.1038/s41598-021-84768-8 (2021).

2. Zhang, X. et al. Reversible loss of core-shell structure for $\mathrm{Ni}-\mathrm{Au}$ bimetallic nanoparticles during $\mathrm{CO} 2$ hydrogenation. Nature Catalysis. 3, 411-417 https://doi.org/10.1038/s41929-020-0440-2 (2020).

3. Shan, A. et al. Atomic-scaled surface engineering Ni-Pt nanoalloys towards enhanced catalytic efficiency for methanol oxidation reaction. Nano Res. 13, 3088-3097 https://doi.org/10.1007/s12274-020-2978-3 (2020).

4. Zhang, X. et al. Pd-Zn nanocrystals for highly efficient formic acid oxidation. Catal. Sci. Technol. 8, 4757-4765 https://doi.org/10.1039/C8CY01503A (2018).

5. Lummen, N. \& Kraska, T. Molecular dynamics investigations of the coalescence of iron clusters embedded in an inert-gas heat bath. Phys. Rev. B. 71, https://doi.org/10.1103/PhysRevB.71.205403 (2005).

6. Mashiach, R. et al. In situ NMR reveals real-time nanocrystal growth evolution via monomerattachment or particle-coalescence. Nature Communications. 12, 229 https://doi.org/10.1038/s41467-020-20512-6 (2021).

7. Lim, T. H. et al. Real-Time TEM and Kinetic Monte Carlo Studies of the Coalescence of Decahedral Gold Nanoparticles. ACS Nano. 3, 3809-3813 https://doi.org/10.1021/nn9012252 (2009).

8. Magdassi, S., Grouchko, M., Berezin, O. \& Kamyshny, A. Triggering the Sintering of Silver Nanoparticles at Room Temperature. ACS Nano. 4, 1943-1948 https://doi.org/10.1021/nn901868t (2010). 
9. Grammatikopoulos, P. et al. Kinetic trapping through coalescence and the formation of patterned AgCu nanoparticles. Nanoscale. 8, 9780-9790 https://doi.org/10.1039/c5nr08256k (2016).

10. Asoro, M. A., Ferreira, P. J. \& Kovar, D. In situ transmission electron microscopy and scanning transmission electron microscopy studies of sintering of Ag and Pt nanoparticles. Acta Mater. 81, 173-183 https://doi.org/10.1016/j.actamat.2014.08.028 (2014).

11. Ding, F., Rosen, A. \& Bolton, K. Size dependence of the coalescence and melting of iron clusters: A molecular-dynamics study. Phys. Rev. B. 70, https://doi.org/10.1103/PhysRevB.70.075416 (2004).

12. Antunez-Garcia, J., Mejia-Rosales, S., Perez-Tijerina, E., Montejano-Carrizales, M., Jose-Yacaman, M. \& J. \& Coalescence and Collisions of Gold Nanoparticles. Materials. 4, 368-379 https://doi.org/10.3390/ma4020368 (2011).

13. Kim, H. Y., Lee, S. H., Kim, H. G., Ryu, J. H. \& Lee, H. M. Molecular dynamic simulation of coalescence between silver and palladium clusters. Mater. Trans. 48, 455-459 https://doi.org/10.2320/matertrans.48.455 (2007).

14. Subbaraman, R. \& Sankaranarayanan, S. K. R. S. Momentum induced coalescence and alloying of Fe-Ni nanoclusters: A molecular dynamics simulation study. Chem. Phys. Lett. 522, 56-61 https://doi.org/10.1016/j.cplett.2011.11.068 (2012).

15. Liu, W., Sun, K. \& Wang, R. M. In situ atom-resolved tracing of element diffusion in NiAu nanospindles. Nanoscale. 5, 5067-5072 https://doi.org/10.1039/c3nr00829k (2013).

16. Ferrando, R., Jellinek, J., Johnston, R. L. \& Nanoalloys From Theory to Applications of Alloy Clusters and Nanoparticles. Chem. Rev. 108, 845-910 https://doi.org/10.1021/cr040090g (2008).

17. Guevara-Chapa, E. \& Mejia-Rosales, S. Molecular dynamics of coalescence and collisions of silver nanoparticles. J. Nanopart. Res. 16, https://doi.org/10.1007/s11051-014-2757-8 (2014).

18. Zhao, Z., Fisher, A. \& Cheng, D. J. Phase diagram and segregation of Ag-Co nanoalloys: insights from theory and simulation. Nanotechnology. 27, https://doi.org/10.1088/0957-4484/27/11/115702 (2016).

19. Wen, Y. H., Li, L., Shao, G. F. \& Huang, R. Thermal Stability of Unary to Quinary Noble-Metal/3dTransition-Metal Alloy Nanoparticles from Molecular Dynamics Simulations: Implications for Multimetallic Catalysis. Acs Applied Nano Materials. 3, 5381-5389 https://doi.org/10.1021/acsanm.0c00746 (2020).

20. Zhou, X. W., Johnson, R. A. \& Wadley, H. N. G. Misfit-energy-increasing dislocations in vapordeposited CoFe/NiFe multilayers. Phys. Rev. B. 69, 144113 (2004).

21. Meng, L. J., Peng, X. Y., Zhang, K. W., Tang, C. \& Zhong, J. X. Structural phase transitions of FeCo and FeNi nanoparticles: A molecular dynamics study. J. Appl. Phys. 111, 024303-024305 (2012).

22. Li, B. Q., Xing, G. Z., Wang, H. L. \& Wang, R. M. Tailoring characteristic thermal stability of Ni-Au binary nanocrystals via structure and composition engineering: theoretical insights into structural evolution and atomic inter-diffusion. AIP Adv. 4, https://doi.org/10.1063/1.4902341 (2014).

23. Plimpton, S. Fast Parallel Algorithms for Short-Range Molecular Dynamics. J. Comput. Phys. 117, 119 https://doi.org/10.1006/jcph.1995.1039 (1995). 
24. Zhao, S. J., Wang, S. Q., Yang, Z. Q. \& Ye, H. Q. Coalescence of three silver nanoclusters: a molecular dynamics study. Journal of Physics-Condensed Matter. 13, 8061-8069 https://doi.org/10.1088/0953-8984/13/35/313 (2001).

25. Miki-Yoshida, M., Tehuacanero, S. \& José-Yacamán, M. On the high temperature coalescence of metallic nanocrystals. Surf. Sci. 274, L569-L576 https://doi.org/10.1016/0039-6028(92)90827-S (1992).

26. Li, G. J. et al. Formation of bcc and fcc during the coalescence of free and supported Fe and $\mathrm{Ni}$ clusters. Phys. Chem. Chem. Phys. 17, 21729-21739 https://doi.org/10.1039/c5cp02929e (2015).

27. Wang, J. D., Chen, S., Cui, K., Li, D. G. \& Chen, D. R. Approach and Coalescence of Gold Nanoparticles Driven by Surface Thermodynamic Fluctuations and Atomic Interaction Forces. ACS Nano. 10, 2893-2902 https://doi.org/10.1021/acsnano.5b08236 (2016).

28. Gutierrez-Wing, C. et al. Coalescence phenomena in 1D silver nanostructures. Journal of PhysicsCondensed Matter. 21, https://doi.org/10.1088/0953-8984/21/29/295301 (2009).

29. Wen, Y. H., Li, L., Zhao, T. \& Huang, R. Solid-Liquid Coexistence in Trimetallic Heterostructured Nanoparticle Catalysts: Insights from Molecular Dynamics Simulations. ACS Applied Nano Materials. 3, 12369-12378 https://doi.org/10.1021/acsanm.0c02862 (2020).

30. Zhao, L., Heinig, N., Leung, K. T. \& One-Step Cooperative Deposition of Au-Ni Alloy Nanoparticles on a Si Substrate: "Catalytic" Growth Mechanism in an Aqueous Medium. J. Phys. Chem. C. 116, 12322-12329 https://doi.org/10.1021/jp302336k (2012).

31. Bondi, J. F. et al. Optimized Synthesis and Magnetic Properties of Intermetallic Au3Fe1 - x, Au3Co1 - x, and Au3Ni1 - x Nanoparticles. Chem. Mater. 22, 3988-3994 https://doi.org/10.1021/cm100705c (2010).

32. Huang, R., Shao, G. F., Zeng, X. M. \& Wen, Y. H. Diverse Melting Modes and Structural Collapse of Hollow Bimetallic Core-Shell Nanoparticles: A Perspective from Molecular Dynamics Simulations. Sci. Rep. 4, 7051 https://doi.org/10.1038/srep07051 (2014).

33. Faccin, G. M., San-Miguel, M. A., Andres, J. \& Longo, E. Silva, E. Z. Computational Modeling for the Ag Nanoparticle Coalescence Process: A Case of Surface Plasmon Resonance. J. Phys. Chem. C. 121, 7030-7036 https://doi.org/10.1021/acs.jpcc.7b00769 (2017). da

34. Yang, L. Q., Gan, Y., Zhang, Y. W. \& Chen, J. K. Molecular dynamics simulation of neck growth in laser sintering of different-sized gold nanoparticles under different heating rates. Applied Physics aMaterials Science \& Processing. 106, 725-735 https://doi.org/10.1007/s00339-011-6680-x (2012).

35. Wanjala, B. N. et al. Nanoscale Alloying, Phase-Segregation, and Core - Shell Evolution of Gold Platinum Nanoparticles and Their Electrocatalytic Effect on Oxygen Reduction Reaction. Chem. Mater. 22, 4282-4294 https://doi.org/10.1021/cm101109e (2010).

36. Teng, X., Shan, A., Yuchen, Z., Wang, R. \& Lau, W. M. Promoting methanol-oxidation-reaction by loading PtNi nano-catalysts on natural graphitic-nano-carbon. Electrochim. Acta. 353, 136542 https://doi.org/10.1016/j.electacta.2020.136542 (2020). 


\section{Figures}

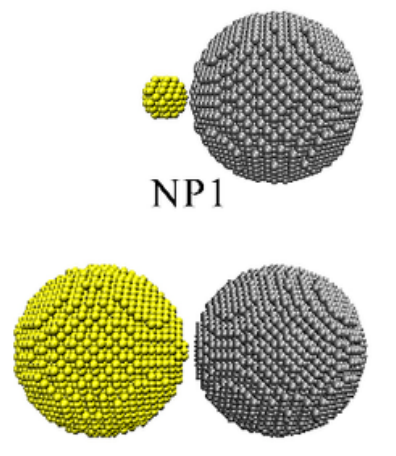

NP5
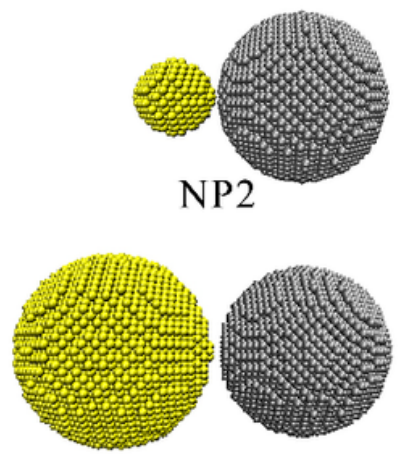

NP6
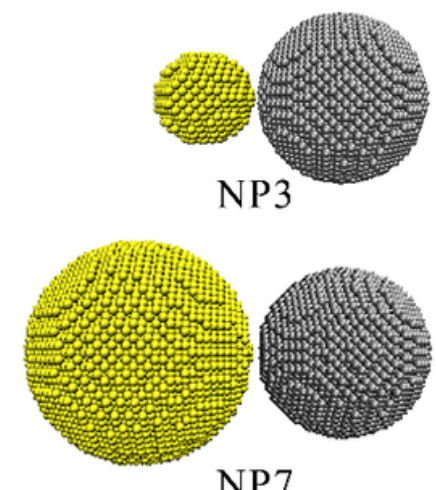

NP7
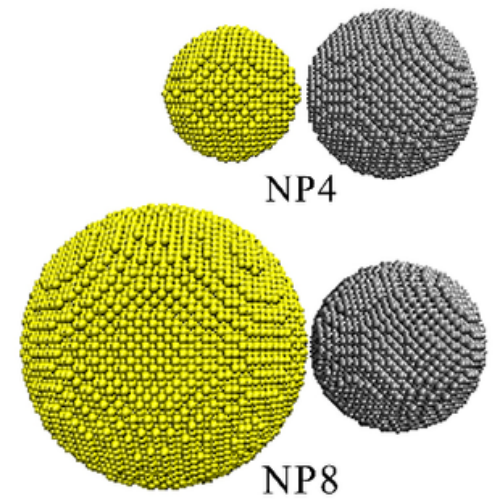

Figure 1

Initial configurations of Ni-Au NPs structures with different atomic ratio, Au and Ni atoms are colored in yellow and gray, respectively.
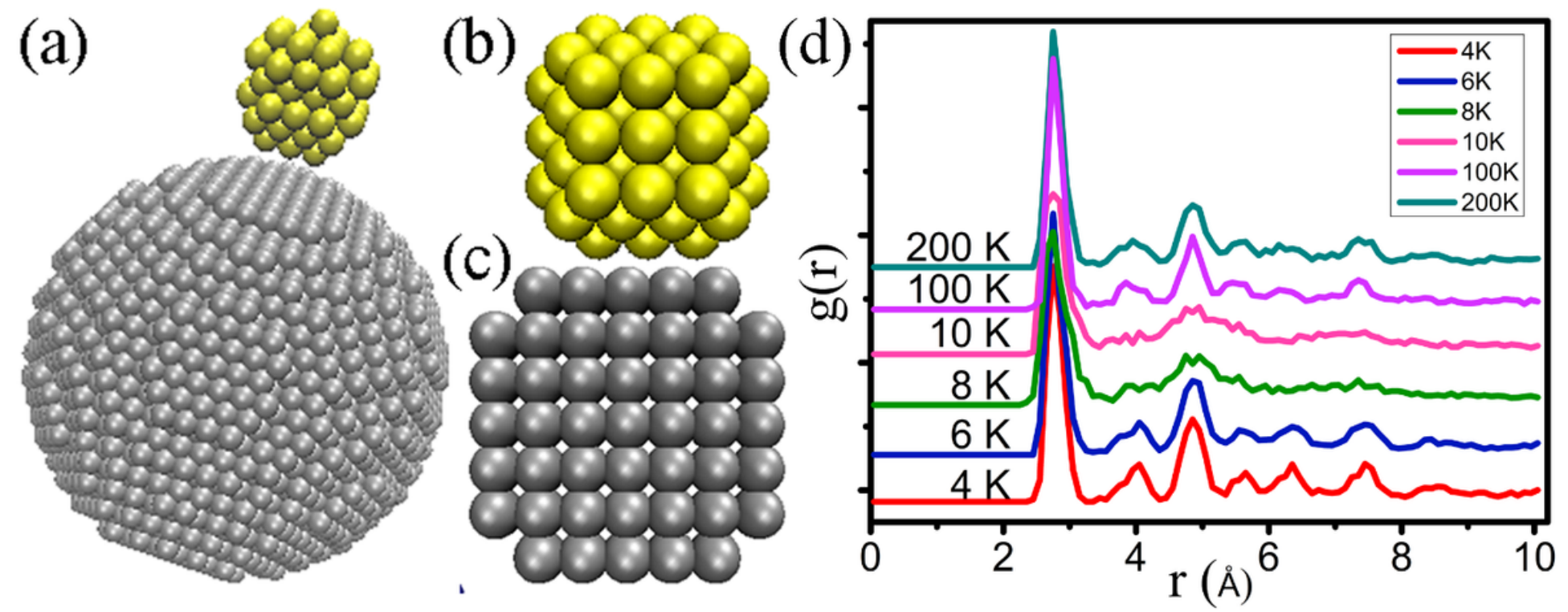

Figure 2

(a) The configurations of the simulated NP1 after a relaxation (b) (100) face of Au atoms (c) (100) face of $\mathrm{Ni}$ atoms (d) PDF of Au atoms in various temperatures. 


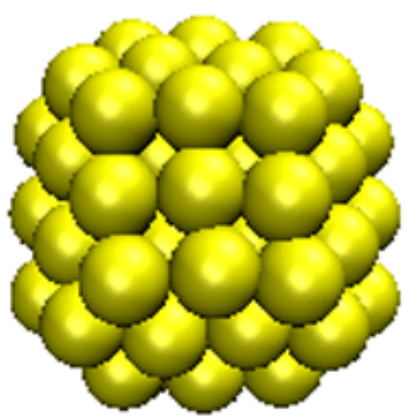

$6 \mathrm{~K}$

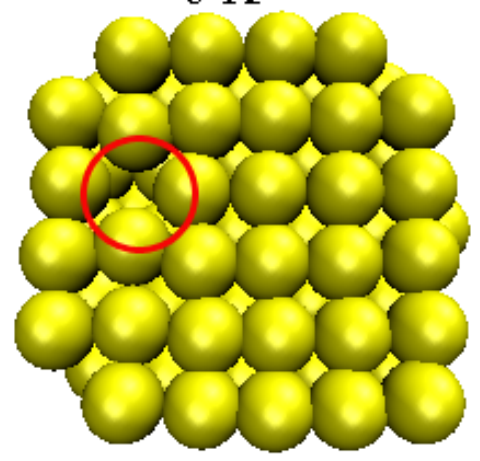

$200 \mathrm{~K}$

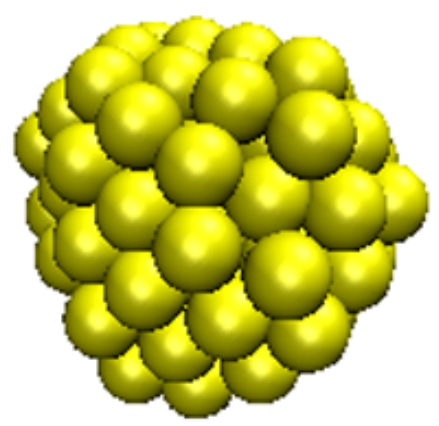

$10 \mathrm{~K}$

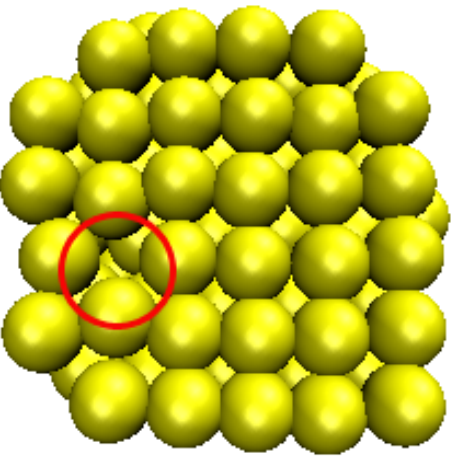

$306 \mathrm{~K}$

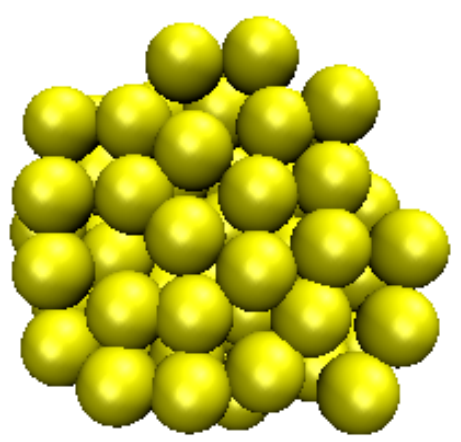

$94 \mathrm{~K}$

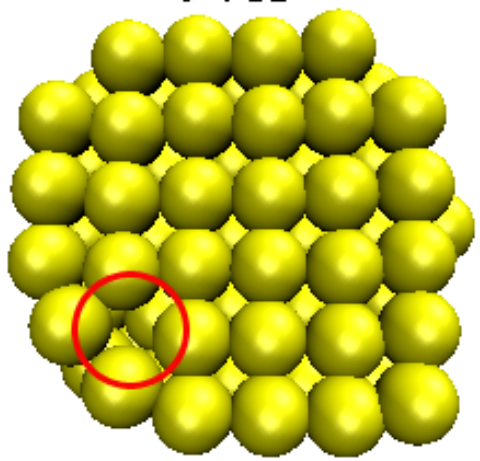

$314 \mathrm{~K}$

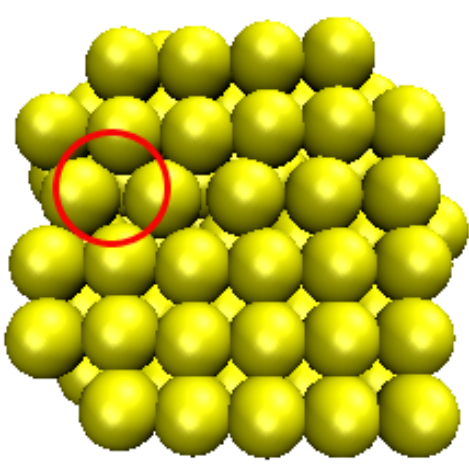

$100 \mathrm{~K}$

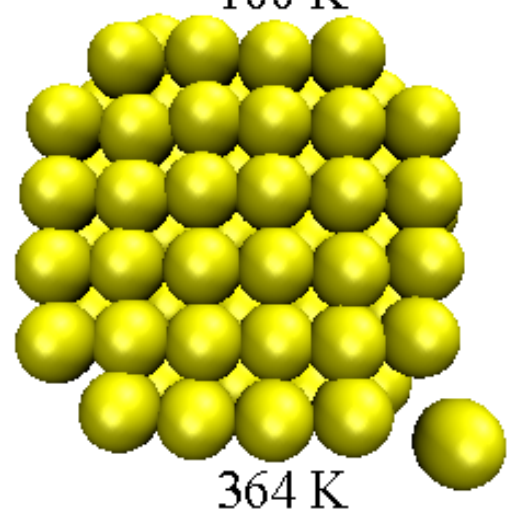

Figure 3

Snapshots of cross sections of Au atoms in the Au-Ni bimetallic NPs taken at eight representative temperatures (unchanged of (100) face of $\mathrm{Ni}$ atoms, as shown in the Fig. 2c). 

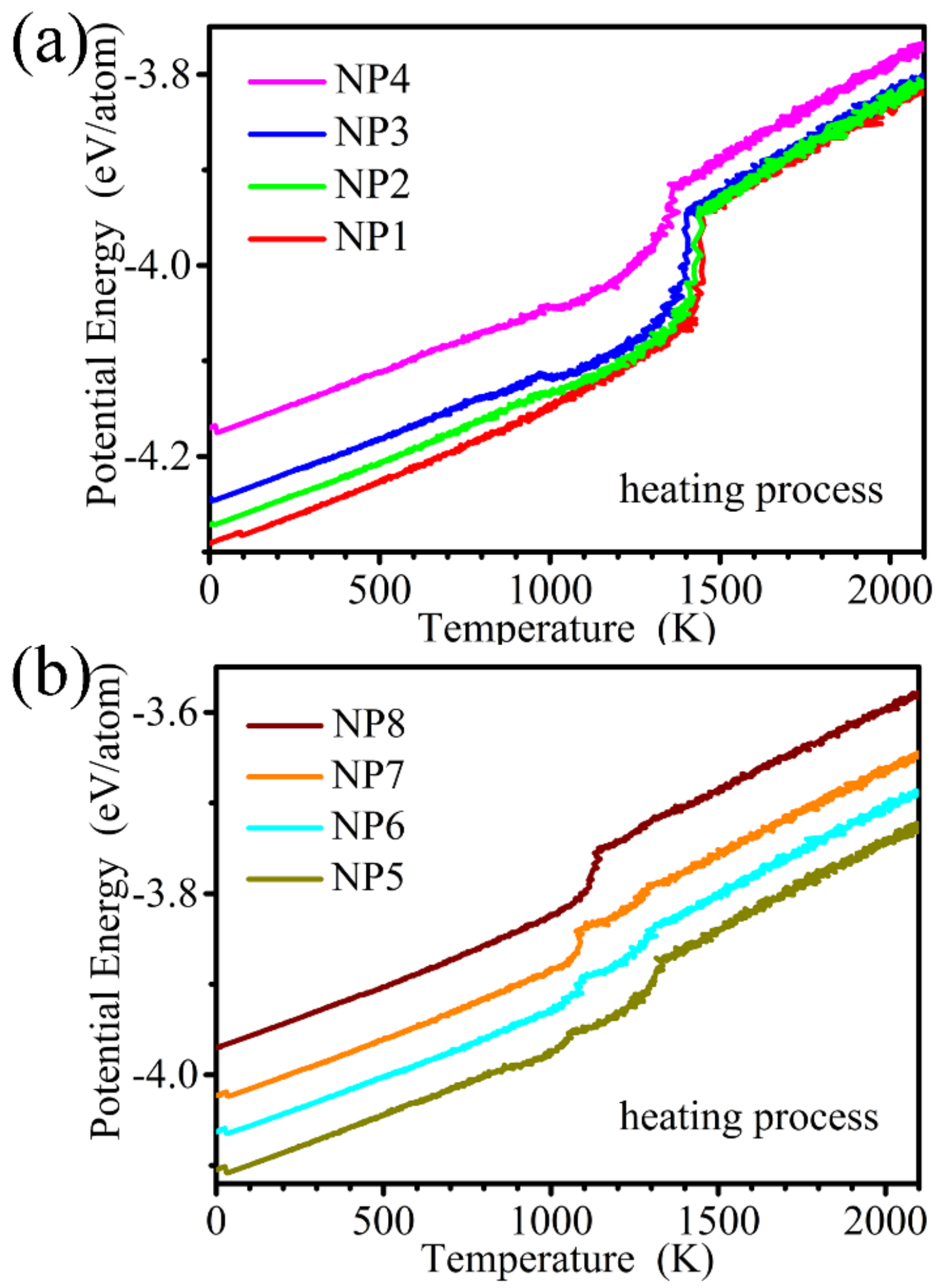

Figure 4

Variation of potential energy as function of temperature for Janus-type NPs heating process (a) NP1, NP2, NP3, NP4 (b) NP5, NP6, NP7, NP8. 
(a)
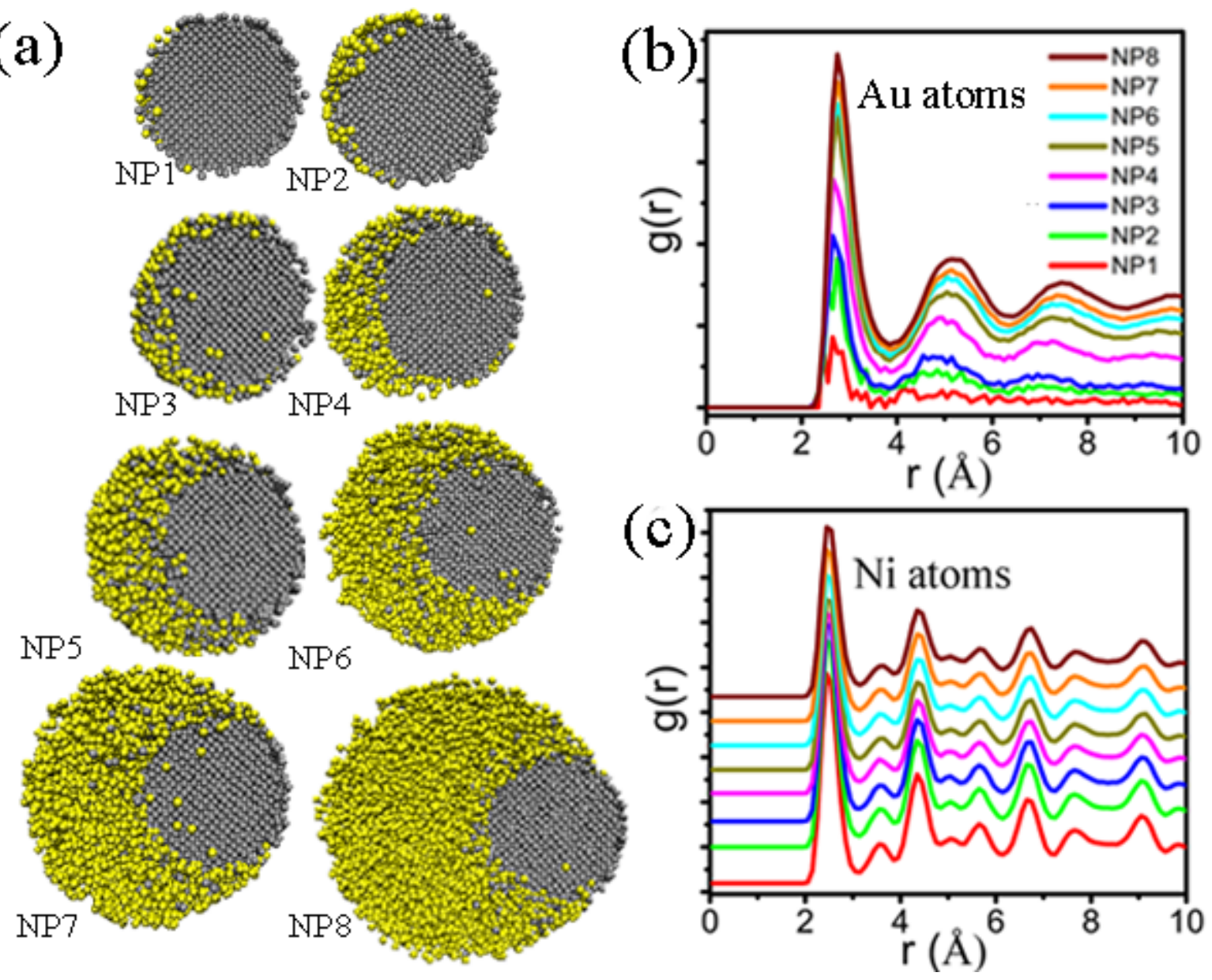

Figure 5

(a)Cross sections through the Au-Ni NPs at temperature 1173 K. (b) and (c) PDF of Au atoms and Ni atoms at temperatures $1173 \mathrm{~K}, \mathrm{Au}$ and $\mathrm{Ni}$ atoms are colored in yellow and gray respectively. 

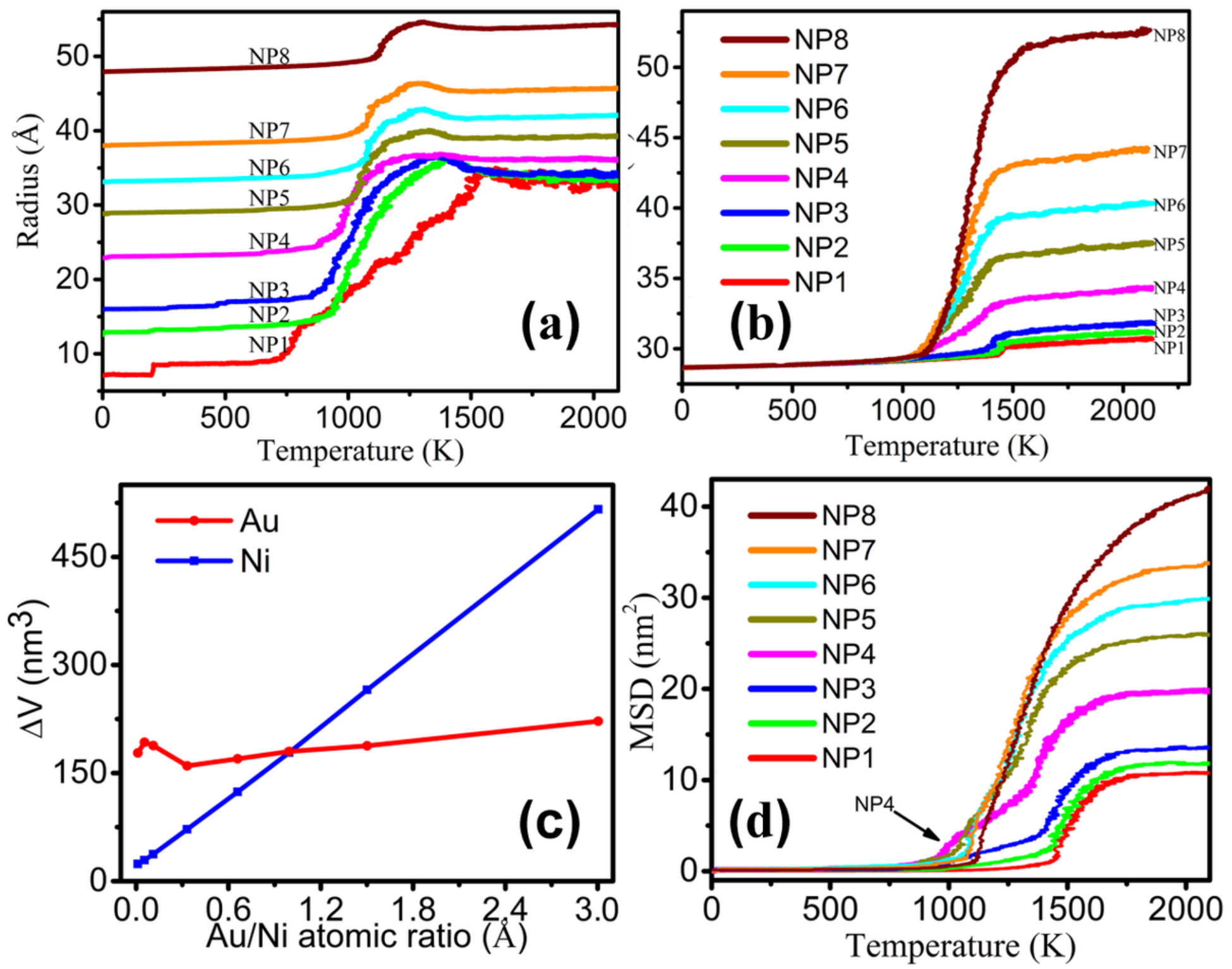

Figure 6

Evolution of radius of Au atoms (a) and Ni atoms (b) during heating process, (c) Volume change versus $\mathrm{Au} / \mathrm{Ni}$ atomic ratio (d) MSD of NP1-NP8. 

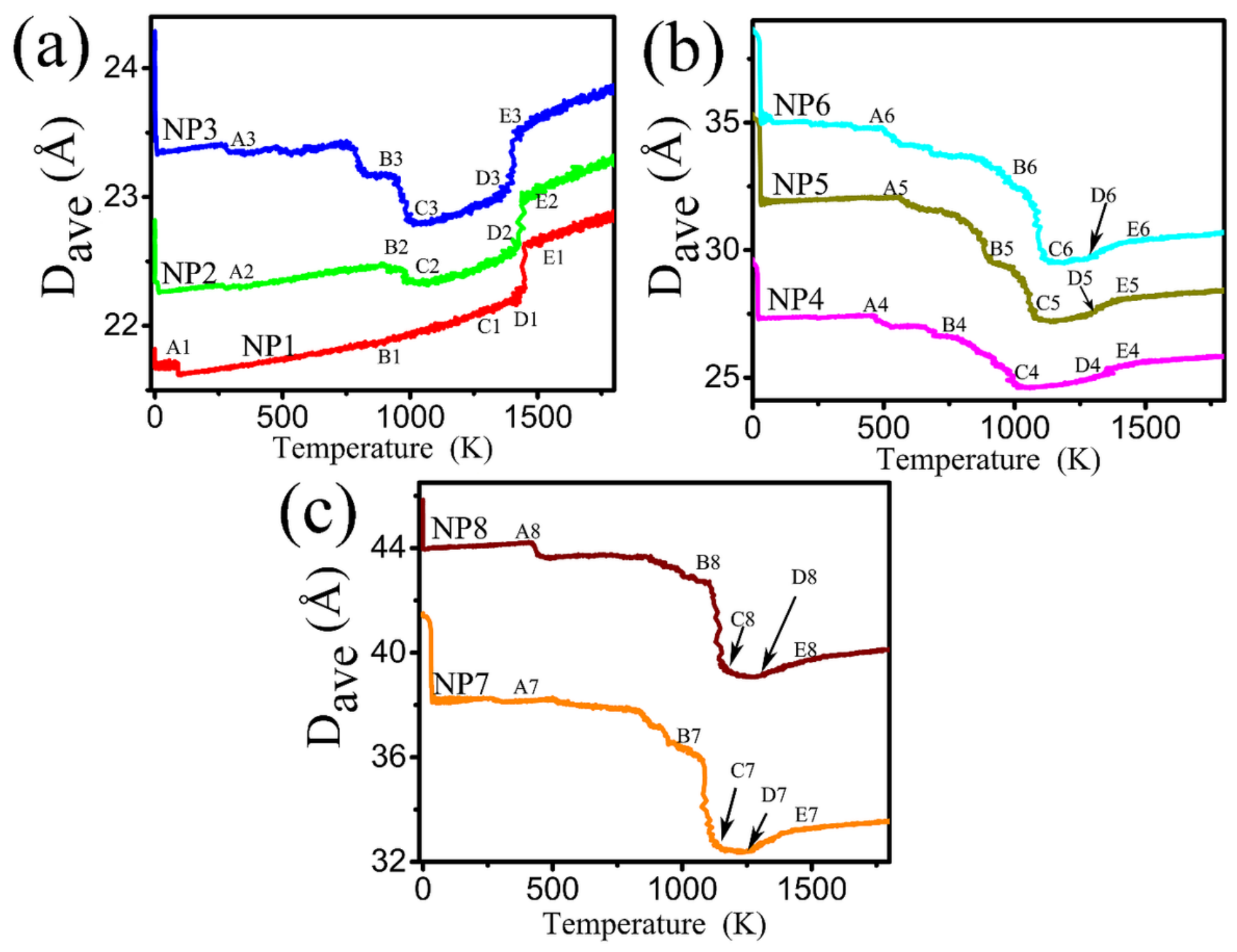

Figure 7

Temperature dependence of Dave, during the coalescence. (a) NP1, NP2, NP3 (b) NP4, NP5, NP6 (c) NP7, NP8.

\section{Supplementary Files}

This is a list of supplementary files associated with this preprint. Click to download.

- ElectronicSupplementaryInformationMDC.docx 